\section{Percepciones de los trabajadores del sector salud frente a Internet y las tecnologías móviles en Colombia}

\author{
José Ignacio Valenzuela, ${ }^{1}$ \\ Juan Camilo Camacho, ${ }^{1}$ \\ Arturo Argüello, ${ }^{2}$ \\ Juan Gabriel Cendales ${ }^{1}$ y \\ Roosevelt Fajardo ${ }^{1}$
}

Forma de citar: Valenzuela JI, Camacho JC, Argüello A, Cendales JG, Fajardo R. Percepciones de los trabajadores del sector salud frente a Internet y las tecnologías móviles en Colombia. Rev Panam Salud Publica. 2009;25(4):367-74.

Palabras clave: tecnología de la información, médicos, teléfono celular, Internet, Colombia.

\footnotetext{
1 Fundación Santa Fe de Bogotá. División de Educación. Bogotá, Colombia. La correspondencia deberá enviarse al doctor José Ignacio Valenzuela, Jefe de Proyectos Especiales, Fundación Santa Fe de Bogotá, División de Educación. Carrera 7b No. 123 - 90, Bogotá, Colombia. T.: 57+1+6030303 Extensión 5721. Correo electrónico: jose.valenzuela@ fsfb.edu.co

2 Departamento de Planeación y Desarrollo Académico. Universidad del Rosario, Bogotá, Colombia.
}

\section{LAS NUEVAS TECNOLOGÍAS}

Internet, la red más grande de computadores del mundo, constituye probablemente el desarrollo más importante de la comunicación global desde el surgimiento de la televisión y del teléfono. Esta red ofrece comunicación de alta velocidad e información versátil, en muchos casos de gran calidad, en un medio económico y fácil de usar. A pesar de la carencia de infraestructura tecnológica y de la barrera impuesta por los bajos índices de conectividad en los países en vías de desarrollo (1), el acceso es universal y el impacto parece ilimitado.

Originalmente concebida para la transmisión de datos en aplicaciones militares, su desarrollo se remonta a la década de 1960. Para ese entonces, se trataba de una red cooperativa de cuatro computadoras localizadas en universidades de los Estados Unidos de América (EUA) - el Instituto de Investigación de Stanford; la Universidad de California en Los ÁngelesUCLA; la Universidad de California en Santa Bárbara, y la Universidad de UTA-, y comenzaba a utilizarse para la transmisión de datos encriptados (2). Más tarde, a principios de la década de los noventa, con la creación de la World Wide Web (WWW) y permitiendo el acceso libre al código HTML, Tim Berners-Lee favoreció el desarrollo de aplicaciones para el público general convirtiendo, tan sólo 50 años después de las primeras pruebas de transmisión de datos, a la red mundial en una herramienta indispensable como medio masivo informativo, educativo, investigativo e interactivo, que cuenta en la actualidad con más de 250 millones de dominios o páginas web en el mundo (2). Desde entonces, cada vez con mayor frecuencia se desarrollan nuevas y mejores formas de comunicación, lo cual ha derivado en una ampliación exponencial de los portafolios de servicios de las distintas instituciones.

El sector salud no ha sido ajeno a esta realidad y ha incorporado paulatinamente tanto Internet como las tecnologías móviles de información y comunicación a la educación y a la prestación de servicios, beneficiando tanto a los profesionales como a los usuarios del sector (3). Internet, además de ser un proveedor constante de información, ha facilitado la difusión de aplicaciones virtuales de alto impacto para la educación, que permiten el acceso global a los medios de aprendizaje a cualquier hora y desde cualquier lugar, eliminando las barreras espacio-temporales propias de los programas presenciales de atención y capacitación. Uno de los desarrollos de mayor alcance es el correspondiente al aprendizaje móvil o m-learning (mobilelearning) que, como su nombre lo indica, utiliza los dispositivos móviles e inalámbricos para complementar la experiencia pedagógica. Los teléfonos celulares, las 
computadoras de bolsillo (como el asistente digital personal —PDA, por su sigla en inglés: personal digital assistant-y los Pocket PC, entre otros) y la Internet, se pueden mezclar en este recurso de manera que se disponga de la información cuando y donde se necesite, optimizando tanto los procesos de enseñanza como la prestación de servicios de salud donde haya conectividad y acceso a tecnologías informáticas, hoy en día consideradas como básicas.

Lo anterior adquiere especial relevancia en países en desarrollo como Colombia, donde cerca de $70 \%$ de la población (alrededor de 30 millones de usuarios) cuentan con acceso a telefonía móvil $(4,5)$ y donde más de $16 \%$ (esto es, una cifra superior a 7 millones de usuarios) tienen acceso a Internet, de los cuales aproximadamente $11 \%$ (750 000 personas) son suscriptores de banda ancha. Este último servicio ha aumentado de manera estrepitosa en los últimos años (incremento superior a $70 \%$ entre los meses de junio de 2006 y 2007) (4), de tal manera que en 2005 se ubicó en el cuarto lugar de crecimiento en el mundo con respecto al número de conexiones con banda ancha, y en el primero en América Latina en cuanto al número de usuarios, con $151 \%$ de incremento respecto al año anterior. En la figura 1 se muestra el crecimiento en la cobertura de los servicios de Internet entre 1995 y 2006 en Colombia $(6,7)$. Por su parte, la telefonía móvil en Colombia ha tenido un crecimiento acelerado en los últimos ocho años, pasando de 2257000 ususarios en 2000 a 30826000 en 2007. En cuanto a la distribución por estrato social, $28 \%$ corresponden a los estratos 1, 2 y 3 , mientras que $72 \%$ se ubican en los estratos 4,5 y 6 (8).

A pesar de todo lo anterior, son pocos los estudios notificados acerca de las actitudes y las percepciones por parte de los trabajadores del sector salud con relación a los nuevos recursos tecnológicos de información y comunicación como Internet, las tecnologías móviles informáticas y sus derivados para la educación (e-learning, m-learning) y para la prestación de

FIGURA 1. Penetración de Internet en Colombia, 1995-2006

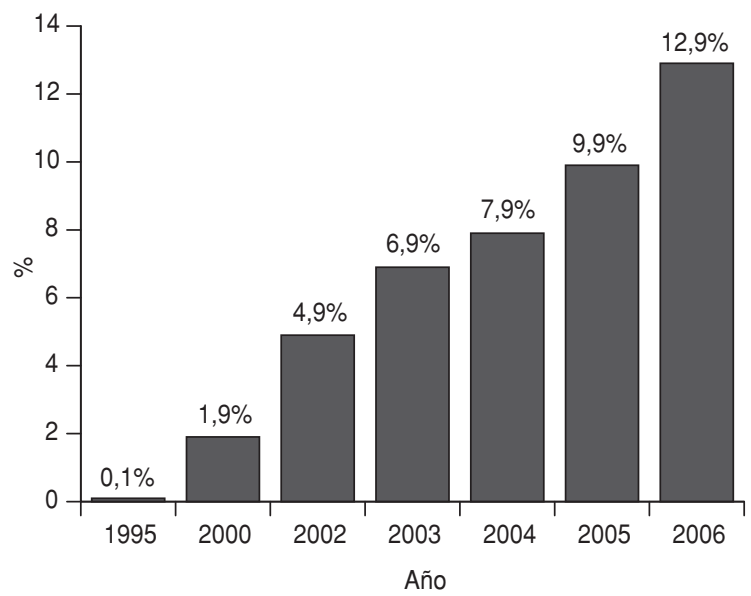

Fuente: Miniwatts Marketing Group. Internet World Stats. [Sitio en Internet] Informe Miniwatts Marketing Group. Hallado en: http://www.internetworldstats.com/ stats.htm. Acceso el 26 marzo 2008. servicios. Algunas publicaciones sugieren un impacto positivo de estos recursos en la capacitación, la atención clínica y la relación médico-paciente (9-15). Cabe destacar un estudio realizado en Dinamarca (16), donde se mostró que cerca de $90 \%$ de los estudiantes de primer año de medicina utilizan el correo electrónico de manera regular, $80 \%$ recurren a Internet para realizar investigación médica y cerca de $60 \%$ tienen acceso a estos servicios desde casa (16). Por otro lado, en otros estudios realizados en grandes cohortes de personal médico se afirma que, aunque constituye un buen recurso, Internet posee mucha información inadecuada que es de fácil acceso para los pacientes (17, 18). De igual manera y de acuerdo con la literatura disponible, a pesar de la difusión del correo electrónico y los servicios de teleconsulta en los EUA, el acceso del público a consultas médicas a través de Internet sigue siendo bajo y no ha demostrado un crecimiento significativo (19). Resulta interesante el hecho de que, hasta la fecha, no haya suficientes trabajos al respecto en los países en vías de desarrollo.

El objetivo del presente estudio es notificar las actitudes y la apreciación de los profesionales del sector salud en cuanto a la incorporación de las tecnologías de la información en su trabajo rutinario, así como realizar una primera aproximación acerca de la opinión de los mismos con relación al uso de las tecnologías móviles.

\section{EL CUESTIONARIO}

\section{Diseño y metodología}

Durante el mes de julio de 2007, durante un evento académico en la ciudad de Medellín, Colombia, se invitó a 1200 profesionales de todas las ramas de la salud a responder un cuestionario estructurado en formato físico de ocho preguntas cerradas con opción de múltiple respuesta.

A través de este instrumento se indagó la edad, el género, la frecuencia de utilización de Internet, el sitio de acceso a la red, la receptividad con respecto a obtener información vía Internet o a través de sistemas móviles y las actitudes en relación con el hecho de que las instituciones de salud utilicen la red mundial y las tecnologías móviles para la prestación de servicios (como consulta de citas médicas, resultados de exámenes diagnósticos y consultas médicas profesionales). Asimismo, el cuestionario inquiría sobre la penetración de la telefonía celular en el sector salud y su posible uso como herramienta de educación.

En total 493 individuos respondieron voluntariamente el instrumento; los resultados se registraron en una base de datos abierta (Open Database Connectivity u ODBC) y se analizaron con el programa Microsoft Excel. Los datos se digitaron en dos ocasiones para evitar errores en la sistematización, revisando que la totalidad de las encuestas hubiesen sido efectivamente digitadas. Más adelante, se depuraron los datos cruzándolos y revisando, mediante verificación automática, los filtros y rangos de valores para cada variable de interés. 


\section{Análisis de la información}

Los datos se examinaron de manera directa sobre el registro de la ODBC. Las respuestas de los sujetos se analizaron de manera seriada y transversal, cuantificando y categorizando el número de respuestas por pregunta, cruzándolas con las variables demográficas. Se excluyeron las preguntas que fueron respondidas de manera inadecuada.

\section{RESULTADOS DE LA ENCUESTA}

\section{Datos generales}

La tasa de respuesta fue de $41 \%$ (493): la edad promedio de los encuestados fue de 31,2 años (figura 2); $55,7 \%$ eran mujeres y $44,3 \%$ varones. Por otra parte, $58,8 \%$ de los participantes eran médicos, mientras que $41.2 \%$ tenían otras ocupaciones dentro del sector salud incluyendo auxiliares de enfermería $(11,3 \%)$, enfermeras $(15 \%)$, estudiantes de medicina o de enfermería $(14,6 \%)$ y terapeutas respiratorios $(0,20 \%)$.

\section{Acceso a Internet}

Del total de sujetos encuestados, $97,6 \%$ refirieron tener acceso a Internet, 60,4\% diario, 27,8\% semanalmente, $4,5 \%$ quincenalmente, $3 \%$ mensualmente, $2,4 \%$ nunca y el 1,8\% restante, con otra frecuencia diferente a las mencionadas. Los puntos de acceso preferidos fueron la casa $(58 \%)$, el lugar de trabajo $(12,5 \%)$ y la combinación de ambos $(9,5 \%)$, acumuló $80 \%$ de los puntos de acceso (figura 3). En el cuadro 1 se muestran las frecuencias y los porcentajes de los puntos de acceso preferidos por los sujetos de estudio. De los encuestados, 98\% manifestaron interés en comunicarse a través de Internet o sistemas móviles con médicos y otros profesionales de la salud para recibir información.
FIGURA 2. Distribución de los encuestados por grupos de edad, Medellín, Colombia, 2007

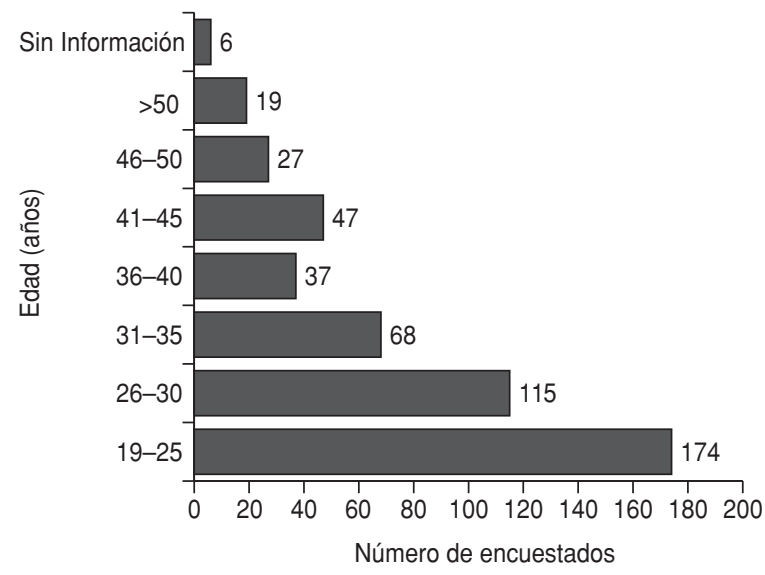

Fuente: Encuesta realizada en julio de 2007 en Medellín, Colombia.

Percepción de Internet y de los sistemas móviles al interior de las instituciones de salud

A través de un cuestionario estructurado, se interrogó a los sujetos participantes acerca de su percepción sobre el uso de Internet por parte de las instituciones prestadoras de servicios de salud (IPSS) para la realización de acciones específicas; $91 \%$ consideraron positivo o muy positivo el hecho de poder verificar su cita médica o el estado de la misma a través de estos servicios. Por otra parte, $90 \%$ opinaron que era positiva la obtención de resultados de exámenes paraclínicos. De los 290 médicos encuestados, en su mayoría menores de 30 años $(60 \%), 256(88,64 \%)$ consideraron como positiva o muy positiva la posibilidad de consultar a un profesional de la salud utilizando Internet (cuadro 2).

FIGURA 3. Frecuencia de acceso a Internet en los trabajadores del sector salud encuestados, Colombia, Medellín, 2007

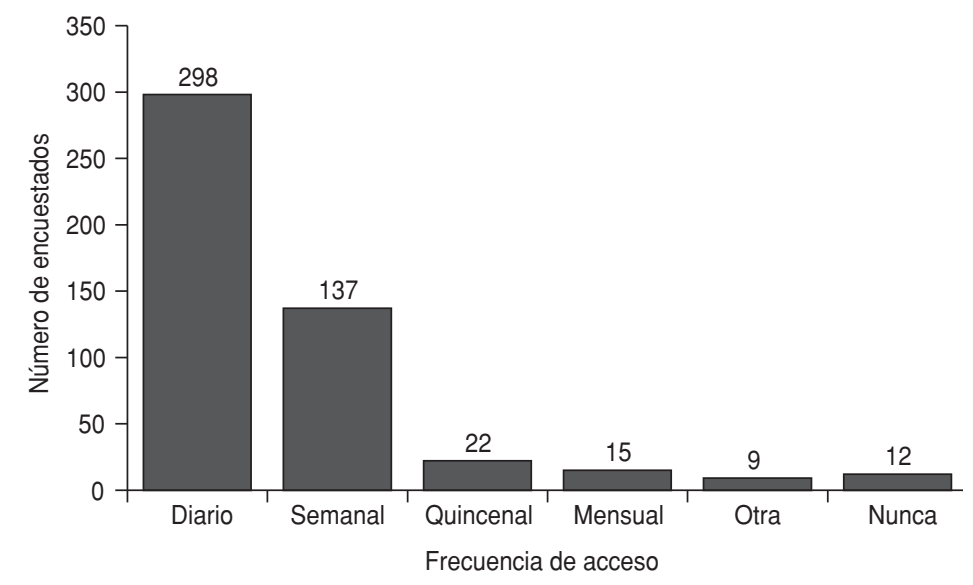

Fuente: Encuesta realizada en julio de 2007 en Medellín, Colombia. 
CUADRO 1. Puntos de acceso a Internet preferidos por trabajadores del sector salud, Colombia, julio 2007

\begin{tabular}{lrr}
\hline \multicolumn{1}{c}{ Lugar de acceso } & Frecuencia & Porcentaje \\
\hline Biblioteca & 4 & 0,81 \\
Celular & 0 & 0,00 \\
Café Internet & 56 & 11,36 \\
Lugar de trabajo & 62 & 12,58 \\
Casa & 289 & 58,62 \\
Café Internet + biblioteca & 1 & 0,20 \\
Casa + biblioteca & 1 & 0,20 \\
Casa + café Internet & 8 & 1,62 \\
Casa + celular & 2 & 0,41 \\
Casa + lugar de trabajo & 47 & 9,53 \\
Lugar de trabajo + café Internet & 4 & 0,81 \\
Otra & 19 & 3,85 \\
Total & 493 & 100,00 \\
\hline Fuente: Encuesta realizada
\end{tabular}

Fuente: Encuesta realizada en julio de 2007, Medellín, Colombia.

En el cuestionario se incluyeron preguntas para conocer las percepciones del uso de Internet y de los sistemas móviles como las PDA o los teléfonos celulares inteligentes para investigación de enfermedades, prestación de servicio al cliente por parte de las IPSS o simplemente como herramienta de investigación. Al respecto, se obtuvieron los siguientes resultados: $80 \%$ consideraron positiva o muy positiva la prestación de dichos servicios a través de Internet, haciendo énfasis en la utilización de sistemas móviles de comunicación; cuando se interrogó acerca del acceso a estos servicios (investigación de enfermedades, prestación de servicio al cliente por parte de las IPSS o simplemente como herramienta de investigación) por medio de dispositivos móviles, se obtuvieron resultados positivos o muy positivos en $80 \%$ de los sujetos encuestados. De igual manera, 83\% del personal médico calificó la prestación de los servicios mencionados como "positivo" o "muy positivo" (cuadro 2).

\section{Telefonía celular}

Del total de encuestados, $98 \%$ manifestaron tener teléfono celular, de ellos, $57,81 \%$ (285) refirieron contar con suscripción a un plan de servicio pospago. Por otra parte, $80 \%$ de los sujetos indicaron estar interesados en el uso de herramientas de educación, gestión e investigación en salud a través de la telefonía celular. Entre el personal médico, $82,41 \%$ (239) de los sujetos manifestaron interés en dichos servicios a través de tecnologías móviles (cuadros 3, 4 y 5). De igual manera, 73\% (54) de las enfermeras encuestadas, 77\% (43) de las auxiliares de enfermería, y 73\% (53) de los estudiantes de medicina o enfermería respondieron afirmativamente en relación con el interés que generan las tecnologías móviles de información para uso como herramienta de educación, gestión e investigación en el sector salud.

\section{CONCLUSIONES}

A pesar de los índices subóptimos de conectividad en Colombia (para el año 2004, 79,8 usuarios por cada 1000 habitantes) (20), se están rompiendo a pasos agigantados las barreras de acceso a un servicio como Internet que debería ser público.

El crecimiento de los usuarios de la red en Colombia ha sido muy importante; así, en lo tocante a los usuarios de banda ancha llegó a 70\% en 2007 con respecto a 2006 (4). Cabe destacar que, en la población encuestada en este estudio, el acceso al servicio es casi universal. El hecho de que los sitios de acceso preferi-

CUADRO 2. Percepción de trabajadores de la salud y de médicos acerca del uso de Internet en las instituciones de salud. Colombia, Julio de 2007

\begin{tabular}{|c|c|c|c|c|c|c|c|c|c|c|c|c|}
\hline & \multicolumn{2}{|c|}{$\begin{array}{l}\text { Citas } \\
\text { médicas }\end{array}$} & \multicolumn{2}{|c|}{$\begin{array}{l}\text { Resultados } \\
\text { de exámenes }\end{array}$} & \multicolumn{2}{|c|}{$\begin{array}{c}\text { Consultar a } \\
\text { profesionales }\end{array}$} & \multicolumn{2}{|c|}{$\begin{array}{l}\text { Investigación } \\
\text { enfermedades }\end{array}$} & \multicolumn{2}{|c|}{$\begin{array}{c}\text { Prestar } \\
\text { mejor servicio }\end{array}$} & \multicolumn{2}{|c|}{$\begin{array}{c}\text { Consulta } \\
\text { información }\end{array}$} \\
\hline & $\mathrm{TS}^{\mathrm{a}}$ & $M^{b}$ & TS & $M$ & TS & $M$ & TS & $M$ & TS & $M$ & TS & $M$ \\
\hline \multicolumn{13}{|l|}{ Internet } \\
\hline Muy positivo & 64,91 & 64,83 & 62,27 & 63,10 & 60,04 & 60,69 & 70,79 & 70,34 & 66,94 & 68,97 & 75,86 & 77,59 \\
\hline Positivo & 25,96 & 25,17 & 27,59 & 27,24 & 28,60 & 27,59 & 22,11 & 23,10 & 25,96 & 24,14 & 18,86 & 16,90 \\
\hline Me da igual & 4,26 & 6,21 & 2,84 & 3,79 & 2,64 & 3,79 & 3,25 & 3,79 & 3,45 & 3,79 & 2,84 & 3,79 \\
\hline Negativo & 0,81 & 0,69 & 3,04 & 2,41 & 3,85 & 3,10 & 0,61 & 0,34 & 0,20 & 0,00 & 0,00 & 0,00 \\
\hline Muy negativo & 0,00 & 0,00 & 0,20 & 0,00 & 0,20 & 0,34 & 0,20 & 0,00 & 0,20 & 0,00 & 0,00 & 0,00 \\
\hline No sabe/No responde & 4,06 & 3,10 & 4,06 & 3,45 & 4,67 & 4,48 & 3,04 & 2,41 & 3,25 & 3,10 & 2,43 & 1,72 \\
\hline Total & 100,00 & 100,00 & 100,00 & 100,00 & 100,00 & 100,00 & 100,00 & 100,00 & 100,00 & 100,00 & 100,00 & 100,00 \\
\hline \multicolumn{13}{|l|}{$\begin{array}{l}\text { Sistemas de } \\
\text { información móvil }\end{array}$} \\
\hline Muy positivo & 57,40 & 55,52 & 51,32 & 50,69 & 51,52 & 50,00 & 53,96 & 52,76 & 56,59 & 56,55 & 58,42 & 56,55 \\
\hline Positivo & 31,24 & 30,69 & 32,66 & 33,79 & 33,27 & 32,41 & 33,67 & 33,79 & 32,86 & 32,07 & 31,03 & 31,03 \\
\hline Me da igual & 5,68 & 7,59 & 5,68 & 6,90 & 5,88 & 7,93 & 5,88 & 7,24 & 5,27 & 6,21 & 5,07 & 6,21 \\
\hline Negativo & 1,62 & 1,72 & 6,49 & 5,86 & 4,67 & 4,83 & 2,84 & 3,10 & 1,83 & 1,72 & 2,43 & 1,72 \\
\hline Muy negativo & 0,20 & 0,34 & 0,61 & 0,34 & 0,61 & 1,03 & 0,41 & 0,69 & 0,41 & 0,69 & 0,81 & 0,69 \\
\hline No sabe/No responde & 3,85 & 4,14 & 3,25 & 2,41 & 4,06 & 3,79 & 3,25 & 2,41 & 3,04 & 2,76 & 2,23 & 2,76 \\
\hline Total & 100,00 & 100,00 & 100,00 & 100,00 & 100,00 & 100,00 & 100,00 & 100,00 & 100,00 & 100,00 & 100,00 & 100,00 \\
\hline
\end{tabular}

Fuente: Encuesta realizada en julio de 2007, Medellín, Colombia.

a Trabajadores de la salud.

${ }^{\mathrm{b}}$ Médicos. 
CUADRO 3. Posesión de celulares: profesionales del sector salud y médicos, ${ }^{\text {a }}$ Colombia, julio 2007

\begin{tabular}{lrrrrr}
\hline & \multicolumn{2}{c}{ Respuesta general } & & \multicolumn{2}{c}{ Respuesta médicos } \\
\cline { 2 - 3 } & No. & & & No. & $\%$ \\
\hline Sí & 484 & 98,17 & & 287 & 98,97 \\
No & 5 & 1,01 & & 2 & 0,69 \\
Ns/Nrb & 4 & 0,81 & & 1 & 0,34 \\
Total & 493 & 100,00 & & 290 & 100,00 \\
\hline
\end{tabular}

Fuente: Encuesta realizada en julio de 2007, Medellín, Colombia.

a Respuestas a la pregunta ¿Tiene usted teléfono celular?

${ }^{b} \mathrm{Ns} / \mathrm{Nr}$ : No sabe, no responde.

CUADRO 4. Pertenencia a planes de servicios de telefonía celular: profesionales del sector salud y médicos, ${ }^{\mathrm{a}}$ Colombia, julio 2007

\begin{tabular}{lcrlcr}
\hline & \multicolumn{2}{c}{ Respuesta general } & & \multicolumn{2}{c}{ Respuesta médicos } \\
\cline { 2 - 3 } \cline { 6 - 6 } & No. & & & No. & $\%$ \\
\hline Prepago & 201 & 41,36 & & 94 & 32,64 \\
Pospago & 285 & 57,81 & & 194 & 66,90 \\
No responde & 7 & 1,42 & & 2 & 0,69 \\
Total & & & & \\
$\quad$ celulares & 486 & 98,58 & & 288 & 99,31 \\
Total & 493 & 100,00 & & 290 & 100,00 \\
\hline
\end{tabular}

Fuente: Encuesta realizada en julio de 2007, Medellín, Colombia.

a Respuestas a la pregunta ¿Cuál es su plan de celular actual?

CUADRO 5. Interés en utilizar el celular en procesos educativos: profesionales de la salud y médicos, ${ }^{a}$ Colombia, julio 2007

\begin{tabular}{lrrrrr}
\hline & \multicolumn{2}{c}{ Respuesta general } & & \multicolumn{2}{c}{ Respuesta médicos } \\
\cline { 2 - 3 } & No. & $\%$ & & No. & $\%$ \\
\hline Sí & 394 & 79,92 & & 239 & 82,41 \\
No & 92 & 18,66 & & 48 & 16,55 \\
Ns $/ \mathrm{Nr}^{\text {b }}$ & 7 & 1,42 & & 3 & 1,03 \\
Total & 493 & 100,00 & & 290 & 100,00 \\
\hline
\end{tabular}

Fuente: Encuesta realizada en julio de 2007, Medellín, Colombia.

a Respuestas a la pregunta ¿Estaría interesado en el uso de herramientas de educación, gestión e investigación a través de un celular?

${ }^{b} \mathrm{Ns} / \mathrm{Nr}$ : No sabe, no responde.

dos a Internet sean el hogar, seguido por el lugar de trabajo, donde los entrevistados invierten la mayoría de su tiempo, podría reflejar el constante interés de los profesionales del sector salud por las nuevas tecnologías, el reconocimiento de su importancia y la necesidad de utilizarlas como una herramienta de información en salud; además, se puede inferir que las han incorporado a su vida cotidiana. Lo anterior adquiere mayor relevancia cuando se considera que los trabajadores del sector salud utilizan el servicio todos los días $\mathrm{o}$, en su defecto, semanalmente, y abre grandes oportunidades para la exploración de las tecnologías de la información y los sistemas móviles orientados al desarrollo de herramientas para la educación, la prestación de servicios de salud y la entrega de información dirigida específicamente al personal médico y paramédico.

Hasta donde se sabe, el presente estudio aporta los primeros datos publicados acerca de la penetración tanto de Internet como de la telefonía celular en el ám- bito de los trabajadores de la salud en Colombia. Es alentadora la receptividad y la actitud de los trabajadores de la salud con respecto a Internet y los sistemas móviles como herramientas para solucionar necesidades de información en salud, y abre un nuevo mercado para el desarrollo, la utilización y el aprovechamiento de estas tecnologías, específicamente en la educación y la prestación de servicios de salud. Es interesante observar que, dentro del gremio médico (subgrupo mayoritario de la población encuestada), existe gran interés por las nuevas tecnologías y por lo que éstas pueden aportar a su trabajo rutinario y a los portafolios de servicios de las instituciones, en particular en cuanto a las tecnologías móviles concierne. No obstante, este estudio refleja la percepción de una muestra importante del sector salud, lo cual plantea importantes interrogantes con respecto al nivel de conocimiento con el que se cuenta en referencia a las nuevas tecnologías basadas en Internet y los sistemas móviles.

Dentro del gremio médico se evidencia una situación muy particular: de acuerdo con los resultados obtenidos y sin distinción de edad, los médicos manifestaron interés y una reacción positiva o muy positiva con respecto a la aplicación de tecnologías como Internet para la prestación de servicios por parte de las IPSS. Llama la atención, sobre todo, la obtención de resultados muy similares en cuanto a las tecnologías móviles (específicamente con la utilización de teléfonos celulares). La situación es replicable en los demás grupos profesionales encuestados, en donde cerca de 70\% de las enfermeras, auxiliares de enfermería y estudiantes de estas carreras manifestaron respuestas positivas o muy positivas.

De manera más específica, al interrogar acerca del área de tecnologías móviles se observa que su penetración dentro de la muestra encuestada es bastante alta: alrededor de 95\% de los sujetos afirmaron poseer un teléfono celular. De igual manera cabe destacar que, dentro de la población objeto de estudio, 57,85\% (285) cuentan con planes pospago; esto contrasta con los informes nacionales sobre la utilización de servicios de telefonía celular, donde se afirma la existencia de escasos seis millones de usuarios en este tipo de planes (entre los poco más de 30 millones que cuentan con el servicio de telefonía celular) (8). Estos dos datos permitirían plantear la hipótesis de que la alta penetración de la telefonía celular en el sector salud puede convertirse tanto en una herramienta fundamental de educación en temas de salud, como en un medio de comunicación versátil para la transmisión de información de impacto individual (p.ej. alertas de variables fisiológicas) y poblacional (p.ej. alertas en salud pública). Inclusive, al analizar otros grupos en cuanto a los tipos de planes de telefonía celular, la tendencia es bastante similar: los planes prepago son superados por los pospago (este fenómeno se observa en auxiliares de enfermería, enfermeras y estudiantes del sector).

Los resultados abren un sinnúmero de posibilidades para el fortalecimiento del campo de la educación móvil y la aplicación global de tecnologías móviles al servicio del sector de salud en Colombia. Como 
punto de partida se puede tomar a los profesionales de la salud, quienes manifestaron un interés muy marcado en el uso de herramientas de educación, gestión e investigación a través de la tecnología celular. No obstante, quedan aún por resolver algunas barreras: aunque la penetración de la tecnología móvil ha mostrado una tendencia alentadora en Colombia, el oligopolio que mantienen los operadores de telefonía celular en el país - actualmente sólo tres empresas manejan la totalidad del mercado en el abastecimiento del servicio de telefonía móvil- limita la aplicación de programas a través de las redes de comunicación móvil celular. Sin embargo, el hecho de que la mayoría de los profesionales de la salud cuenten con planes pospago puede abrir las posibilidades de negociación en cuanto a la transmisión de datos, como por ejemplo a través de planes de mensajes SMS (mensajes cortos de texto) ilimitados o del soporte de plataformas que permitan el inicio de programas educativos y de servicios con el sector de la salud. Asimismo, las posibilidades son infinitas ya que los resultados aquí presentados permiten plantear la conformación de una comunidad futura con redes de banda ancha como WiMax para los trabajadores del sector, o redes de acceso libre a Internet con cobertura global como Red FON. En un futuro un poco más lejano, podría incluso pensarse en el establecimiento de sistemas estructurados de teleconsulta o sistemas de segunda opinión con transmisión de datos, voz y video, a través de la explotación de tecnologías móviles ya existentes como 3G (telefonía móvil de "tercera generación"). Mientras tanto, es conveniente aprovechar las bondades y los recursos de las tecnología móviles e informáticas ya disponibles para impactar positivamente el sector salud, como los proyectos para la difusión de información en salud Pubmed (www.pubmed.com), Pubmed Central (http://www.pubmedcentral.nih.gov/), Bireme (http://www.bireme.org/php/index.php), Biomedcentral (http://www.biomedcentral.com/), los desarrollos para tecnología móvil de la National Library of Medicine de los EUA (http://www.nlm.nih. gov/mobile/), los vademécum y sistemas de información medicamentosa para móviles (http://www. epocrates.com/) y las calculadoras médicas para el cálculo automático de índices metabólicos como el de masa corporal, o respiratorios, entre otros (http:/ /www. users.med.cornell.edu/ spon/picu/calc/medcalc. htm, http://www.skyscape.com/EStore/ProductDetail. aspx?ProductID=227, http://www.qxmd.com/).

Aunque algunas limitantes siguen pendientes de resolver - la mayoría de los desarrollos no están disponibles en español, muchos son costosos para el usuario final y algunos no responden ni a las necesidades ni a los recursos de Latinoamérica-, resulta alentador saber que ya se adelantan proyectos colaborativos en la Región para el proyecto e-FORSES (Fortalecimiento de Servicios Esenciales de Salud en Latinoamérica Mediante el Uso de las Tecnologías de la Información y Comunicación) para la sistematización de un aplicativo de historia clínica electrónica en código abierto, interconectada con un sistema para móviles de consulta de información clínica, llevado a cabo por la Fundación Santa Fe de Bogotá (Colombia) y la Universidad Austral (Argentina), dentro del marco del proyecto internacional PESCA (Plataforma para la e-Salud en Código Abierto). Este tipo de iniciativas, apoyadas por las políticas internacionales de expansión de conectividad, pretenden estrechar cada vez más la denominada brecha digital hacia un perfeccionamiento del Gobierno digital (e-Gobierno) en el que los actores de los sistemas de salud puedan trans-accionar de manera recíproca con los demás miembros del Estado para mejorar la eficiencia interna y la provisión de servicios. Asimismo, este tipo de iniciativas llevan al crecimiento de la actual sociedad de la información, donde la generación y el libre intercambio de la información forman parte de todos los procesos productivos, habilitan la inserción en los mercados globales, estimulan la reducción de costos y permiten la adaptación a las cambiantes condiciones del mercado, todo lo cual deriva, necesariamente, en beneficios y ventajas para el mercado imperfecto de la salud.

En países desarrollados, específicamente en los EUA, los ensayos clínicos aleatorizados han planteado la posibilidad de utilizar Internet como un recurso para incrementar la eficiencia en la prestación de servicios de salud. En un estudio cuantitativo realizado en 2003 (21), cuyo objetivo era comprender la experiencia del personal médico con el empleo de las tecnologías de la información y la comunicación, se evidenció la amplia aceptación y el favoritismo hacia éstas en relación con el uso del correo electrónico como medio de comunicación con sus pacientes. No obstante, ese mismo año Katz y colaboradores (22) realizaron un ensayo aleatorizado cuyo objetivo era disminuir la tasa de inasistencia a las citas médicas, mejorar la comunicación con los pacientes y disminuir el número de llamadas telefónicas a los centros de atención médica. Para tal fin, se aleatorizó en dos grupos a los médicos que trabajan en dichos centros de atención: el primero se encargaría de incentivar a sus pacientes en el uso del sistema de correo electrónico, mientras que el segundo, o grupo control, continuaría utilizando el antiguo sistema telefónico. En dicho estudio, el volumen de correo electrónico utilizado no superó de la manera esperada al volumen de llamadas telefónicas y se llegó a la conclusión de que un sistema de clasificación (triage) a través de correo electrónico no sustituye las llamadas telefónicas por parte de los usuarios. Aunque éste no constituye un informe alentador sobre el uso de las tecnologías de la información y la comunicación, otros estudios contradicen esta conclusión. En un trabajo similar llevado a cabo en 2004, White y colaboradores (23) encontraron diferencias significativas a favor del sistema que utiliza la comunicación vía correo electrónico, sobre la telefónica, para el establecimiento de citas y seguimiento de los pacientes. Houston y colaboradores (24), también en 2004, decidieron explorar la experiencia de los pacientes que usaban con frecuencia el servicio de correo electrónico para comunicarse con su médico; recolectaron datos de 1881 individuos de los cuales 16,5\% utilizaban ac- 
tivamente el servicio y catalogaron como eficiente la comunicación a través de este medio para el seguimiento clínico en cuanto a la obtención de información específica en salud. En resumen, la evidencia actual es limitada y contradictoria, y es necesario ampliar la evidencia con respecto a la utilidad de la aplicación de las tecnologías de la información y la comunicación en el sector salud. Con la tendencia creciente de los índices de conectividad en todo el mundo, y a medida que las aplicaciones tecnológicas en el campo de la salud sean incorporadas a la práctica rutinaria, se espera que la utilización de los servicios de salud electrónica se incremente de manera proporcional. De hecho, ya existen guías de buenas prácticas para la redacción de mensajes y correos electrónicos para pacientes (25), lo cual abre el espectro a un sinnúmero de posibilidades de aplicación tanto en Colombia como en otros países en desarrollo, como pueden ser las comunidades virtuales, el establecimiento de citas a través de la red o el informe y seguimiento de procesos clínicos y paraclínicos de los pacientes.

En el contexto colombiano, la alta penetración de estas tecnologías, especialmente del celular, permite aspirar con un considerable grado de realismo a un sistema de salud con una mejor comunicación con los usuarios, con pacientes bien informados, a través de la implementación de estrategias de promoción de la salud basadas en las nuevas tecnologías informáticas y los sistemas móviles de transmisión de datos. A partir de los resultados del presente trabajo, se puede proponer la incorporación de las tecnologías de la información y la comunicación - como Internet y los sistemas móviles - al portafolio de servicios de las instituciones de salud colombianas destacando que, en la muestra encuestada, la mayoría de los trabajadores del sector se mostraron interesados en su aplicación a la práctica rutinaria para verificar estados de citas médicas, revisar exámenes paraclínicos, realizar consultas especializadas no urgentes, o simplemente como herramienta para mejorar el servicio al cliente o para mantener informados a sus usuarios (26). La anterior afirmación invita a las instituciones de salud de Colombia a modernizarse invirtiendo en dichas tecnologías -incluyendo las móviles- para, de esa manera, mejorar la atención y la calidad de los servicios que ofrecen. De igual forma, abre la puerta a la investigación en tecnologías aplicadas a la salud en Colombia.

Si bien este trabajo brinda una primera aproximación a la percepción y potencialidad de la Internet y las tecnologías móviles aplicadas al sector salud, resulta clara la necesidad de llevar a cabo estudios para evaluar el impacto de los esfuerzos y de la inversión en tecnologías en salud, especialmente en el campo de la educación virtual y la prestación de servicios a distancia, tomando en cuenta la costo-efectividad, el impacto clínico y el impacto sobre la salud pública.

\section{LIMITACIONES DEL ESTUDIO}

Este estudio se basa en la aplicación de una encuesta cuyo único objetivo era conocer la percepción de los miembros del sector salud sobre la implementación de nuevas tecnologías, especialmente de los sistemas móviles, y su utilización en el portafolio de servicios de las instituciones del sector. Los elementos de análisis son subjetivos y no permiten establecer conclusiones fehacientes; sin embargo, los datos permiten realizar las primeras aproximaciones e hipótesis que pueden ser evaluadas en futuros estudios.

Una limitante del estudio es que no explica la naturaleza de la buena receptividad del personal de la salud encuestado frente a Internet y los sistemas de comunicación móvil; no es claro si se debe al hecho de que realmente conoce a profundidad los potenciales y beneficios que se pueden derivar de esos medios, o bien porque la propuesta puede parecer atractiva por lo innovadora que resulta en este medio.

Agradecimientos. Se agradece al equipo de trabajo de la División de Educación de la Fundación Santa Fe de Bogotá el haber facilitado los recursos para la elaboración del presente manuscrito. De igual manera, se agradece la colaboración de los participantes y de las siguientes entidades organizadoras del simposio "Urgente saber de Urgencias", llevado a cabo en julio de 2007 en la ciudad de Medellín, Colombia: Fundación Santa Fe de Bogotá; Asociación Colombiana de Hospitales y Clínicas; Hospital Universitario San Vicente de Paul; Hospital Universitario San Ignacio y Fundación Cardiovascular de Colombia.

\section{SYNOPSIS}

\section{Health care workers' perception of the Internet and mobile technologies in Colombia}

In July 2007 in Medellin, Colombia, 1200 health care professionals were asked to complete a questionnaire: of the 493 who participated, the mean age was 31.2 years; $58.8 \%$ were physicians; and $97.6 \%$ had Internet access, $60.5 \%$ on a daily basis and $27.7 \%$, weekly. The preferred place to access the Internet was from home (58\%) or from the work place $(12.5 \%) ; 98 \%$ reported having a cell phone, and of those, $80 \%$ were interested in using health education tools via cell phone. These are the first data published regarding Internet and cellular phone penetration among health care workers in Colombia. Acceptance of the Internet and mobile systems as health information tools is gaining, and as such, creating a new opportunity for training and harnessing of these new technologies.

Key words: Information technology, physicians, cellular phone, Internet, Colombia. 


\section{REFERENCIAS}

1. Al-Shahi R, Sadler M, Rees G, Bateman D. The internet. J Neurol Neurosurg Psychiatry. 2002;73(6):619-28.

2. Paul J, Seib R, Prescott T. The Internet and clinical trials: background, online resources, examples and issues. J Med Internet Res. 2005;7(1):e5.

3. Edejer TT. Disseminating health information in developing countries: the role of the internet. BMJ. 2000;321(7264): 797-800.

4. Ministerio de Comunicaciones de Colombia. Informe Telecomunicaciones 2007. [Sitio en Internet] Informe Ministerio de Comunicaciones de Colombia. Hallado en: http://www.mincomunicaciones.gov. $\mathrm{co} / \mathrm{mincom} / \mathrm{src} /$ user_docs/Archivos/ Documentos/InformeTMC2007.pdf. Acceso el 7 agosto 2007.

5. Departamento Administrativo Nacional de Estadística (DANE). Censo Poblacional Colombia 2005. [Sitio en Internet] Censo DANE. Hallado en: http:/ / www. dane.gov.co/censo. Acceso el 27 agosto 2005.

6. Miniwatts Marketing Group. Internet World Stats. [Sitio en Internet] Informe Miniwatts Marketing Group. Hallado en: http://www.internetworldstats.com/ stats.htm. Acceso el 26 marzo 2008.

7. Comisión de Regulación de Telecomunicaciones. Reporte de Internet en Colombia: Informe consolidado resolución 502/ 02. [Sitio en Internet] Informe Comisión de Regulación de Telecomunicaciones Hallado en: http://www.webcitation. org/5OIRXiaA1. Acceso el 12 marzo 2007.

8. Asociación de la Industria Celular de Colombia (ASOCEL). Estadísticas 2008. [Sitio en Internet] Informe ASOCEL. Hallado en: http://www.asocel.org.co/ pdf/creciento_movil_porcentaje_90.pdf. Acceso el 26 marzo 2008.

9. Ahmad F, Hudak PL, Bercovitz K, Hollenberg E, Levinson W. Are physicians ready for patients with Internet-based health information? J Med Internet Res. 2006;8(3):e22.

10. Farrell MJ, Rose L. Use of mobile handheld computers in clinical nursing education. J Nurs Educ. 2008;47(1):13-9.

11. Waran V, Selladurai BM, Bahuri NF, George GJ, Lim GP, Khine M. Teleconferencing using multimedia messaging service (MMS) for long-range consultation of patients with neurosurgical problems in an acute situation. J Trauma. 2008;64(2):362-5.

12. Kollmann A, Riedl $M$, Kastner $P$, Schreier G, Ludvik B. Feasibility of a mobile phone-based data service for functional insulin treatment of type 1 diabetes mellitus patients. J Med Internet Res. 2007;9(5):e36.

13. Dwivedi A, Wickramasinghe N, Bali RK, Naguib RN. Critical success factors for achieving superior $m$-health success. Int J Electron Healthc. 2007;3(2):261-78.

14. Baldwin LP, Low PH, Picton C, Young T. The use of mobile devices for information sharing in a technology-supported model of care in A\&E. Int J Electron Healthc. 2007;3(1):90-106.

15. Merrell RC, Cone SW, Rafiq A. The authority and utility of internet information. Stud Health Technol Inform. 2008; 131:265-72.

16. Dorup J. Experience and attitudes towards information technology among first-year medical students in Denmark: longitudinal questionnaire survey. J Med Internet Res. 2004;6(1):e10.

17. Murray E, Lo B, Pollack L, Donelan K, Catania J, Lee K, et al. The impact of health information on the Internet on health care and the physician-patient relationship: national U.S. survey among 1.050 U.S. physicians. J Med Internet Res. 2003;5(3):e17.

18. Baker L, Wagner TH, Singer S, Bundorf MK. Use of the Internet and e-mail for health care information: results from a national survey. JAMA. 2003;289(18): 2400-6.

19. Sciamanna $\mathrm{CN}$, Rogers ML, Shenassa ED, Houston TK. Patient access to U.S. physicians who conduct internet or email consults. J Gen Intern Med. 2007; 22(3):378-81.

20. Instituto para la Conectividad de las Américas. Mapa de Conectividad. [Sitio en Internet] Mapa Instituto para la Conectividad de las Américas. Hallado en: http://www.icamericas.net/Map/ mapa/mapagene.html. Acceso el 27 agosto 2007.

21. Patt MR, Houston TK, Jenckes MW, Sands DZ, Ford DE. Doctors who are using e-mail with their patients: a qualitative exploration. J Med Internet Res. 2003;5(2):e9.

22. Katz SJ, Moyer CA, Cox DT, Stern DT. Effect of a triage-based E-mail system on clinic resource use and patient and physician satisfaction in primary care: a randomized controlled trial. J Gen Intern Med. 2003;18(9):736-44.

23. White CB, Moyer CA, Stern DT, Katz SJ. A content analysis of e-mail communication between patients and their providers: patients get the message. J Am Med Inform Assoc. 2004;11(4):260-7.

24. Houston TK, Sands DZ, Jenckes MW, Ford DE. Experiences of patients who were early adopters of electronic communication with their physician: satisfaction, benefits, and concerns. Am J Manag Care. 2004;10(9):601-8.

25. Greiver M. Practice tips. E-mailing patients. Can Fam Physician. 2006;52(9): 1074.

26. Valenzuela JI, Arguello A, Cendales JG, Rizo CA. Web-based asynchronous teleconsulting for consumers in Colombia: a case study. J Med Internet Res. 2007;9(4): e33. 